Rechtsmedizin $2022 \cdot 32: 217-220$ https://doi.org/10.1007/s00194-021-00545-x Angenommen: 27. Oktober 2021 Online publiziert: 3. Dezember 2021 ๑) Der/die Autor(en) 2021

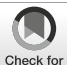

\section{Vitale anale Tierfraßverletzung durch einen Dackel}

\author{
Inga Duval · Elke Doberentz · Burkhard Madea \\ Institut für Rechtsmedizin, Universitätsklinikum Bonn, Bonn, Deutschland
}

\title{
Zusammenfassung
}

Hundebissverletzungen weisen bei Lebenden in der Regel eine charakteristische Morphologie auf, anhand derer sich die Entstehung gut nachvollziehen lässt. Haben Hunde Zugang zu einer Leiche, so kann es zu postmortalen Tierfraßverletzungen kommen, und das Verletzungsbild kann u.U. auf den ersten Blick auch als Fremdbeibringung durch Dritte missinterpretiert werden.

Vorgestellt wird ein seltener Fall einer Tierfraßverletzung an einer lebenden Person. Eine Mischintoxikation mit Zopiclon und Alkohol im häuslichen Umfeld führte zur Bewusstlosigkeit, die einen ausgedehnten Gewebsdefekt im Analbereich durch Bissverletzungen des eigenen Dackels ermöglichte. Außergewöhnlich ist hierbei neben der vitalen Entstehung auch die Lokalisation der Verletzung.

\section{Schlüsselwörter}

Hundebiss · Tierfraß · Bissverletzung · Analtrauma · Zopiclon

\section{Einleitung}

Bissverletzungen durch Hunde werden in Deutschland nicht bundeseinheitlich statistisch erfasst, Schätzungen gehen jedoch von ca. 35.000 Hundebissverletzungen an Menschen pro Jahr aus. In den meisten Fällen ist der beißende Hund den Geschädigten bekannt; häufig handelt es sich sogar um den Hund aus dem eigenen Haushalt

\section{Falldarstellung \\ Vorgeschichte}

Eine 74-jährige Frau war von ihrem Ehemann morgens bewusstlos in ihrem Schlafzimmer aufgefunden worden, nachdem er sie am Vorabend zuletzt gesehen hatte. Beide schliefen getrennt in unterschiedlichen Räumen. Bei Auffindung lag sie in einer Linksseitenlage auf dem Fußboden ihres Schlafzimmers und ihr Unterleib war unbekleidet. Der hinzugerufene Notarzt konnte die Frau nur auf starke Schmerzreize hin erwecken, sie konnte jedoch angeben, dass sie in suizidaler Absicht Schlaftabletten eingenommen hätte. Es wurde eine anale faustgroße, leicht blutende Wunde festgestellt, die von den behandelnden
Ärzten gegenüber der Polizei als „wie ausgeschnitten" beschrieben wurde. Zur Verletzungsentstehung konnte die Frau keine Angaben machen.

Bei der Auffindung der Geschädigten hatte sich der Dackel des Ehepaars mit im Raum befunden. Die Zimmertüre des Raums, wo die Geschädigte aufgefunden wurde, stand offen, sodass der Hund auch Zugang zu den benachbarten Räumlichkeiten gehabt hatte. Auf dem Fußboden waren blut- und stuhlsuspekte Substanzen festgestellt worden. Die Polizei stellte bei einer Nachschau eine Schachtel mit mehreren angetrockneten und mit Fäkalien behafteten Gewebeteilen fest, die der Ehemann zwischenzeitlich vom Fußboden aufgelesen hatte.

An Vorerkrankungen waren bei der Geschädigten eine Leberzirrhose und Alkoholabusus bekannt.

\section{Klinischer Befund}

Bei den klinischen Untersuchungen zeigte sich ein bis tief in das Becken reichender Weichgewebsdefekt mit Amputation des Afters, des Analsphinkters und Teilen des Dickdarms. 


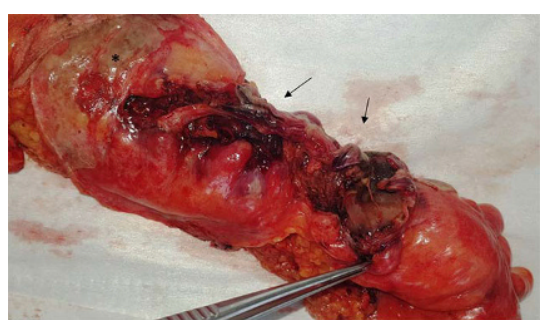

Abb. 1 \ Kolonresektat. Asteriskus: fibrinöse Beläge. Pfeile: eingeblutete fransige Einrisse der äußeren Darmwand und des Mesenteriums. (Quelle: Helios Klinikum Siegburg)

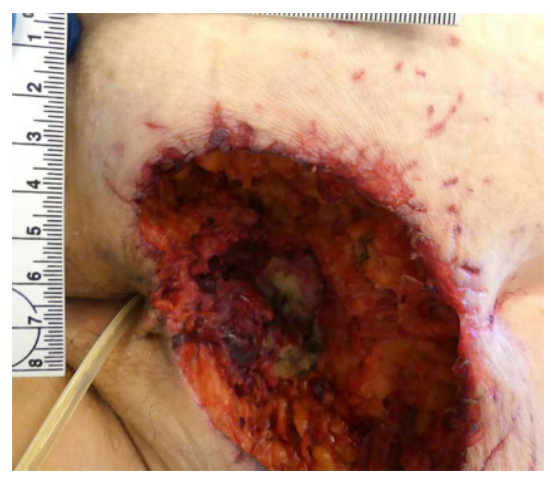

Abb. 4 ム Postoperativer Befund, Wundränder im Detail

Bei ausgedehnter kotiger und gangränöser Peritonitis und paralytischem lleus erfolgte eine Laparotomie mit Rektumexstirpation und Anlage eines Kolostomas. Der ca. $30 \mathrm{~cm}$ lange, resezierte Abschnitt des Dickdarms wies fetzige Zerreißungen der Darmwand und des Mesenteriums auf (• Abb. 1). Die innere Darmschleimhaut wurde von den Operateuren als "wie abgeschmirgelt" beschrieben.

Eine rechtsmedizinische Untersuchung wurde ca. 2,5 Tage nach der Krankenhausaufnahme von der Kriminalpolizei beauftragt. Es fand sich ein rundlicher, ca. $10 \mathrm{~cm}$ durchmessender, klaffender Haut- und Weichgewebedefekt zwischen Scheideneingang und Steißbein, der weit nach innen in Richtung Beckenboden reichte. After oder ein Darmlumen waren nicht zu identifizieren, die Scheidenhinterwand lag frei (ब Abb. 2 und 3 ).

Die Wundränder wiesen eine unregelmäßige Kontur auf, mit zahlreichen fransigen und kratzerartigen Einkerbungen und flächigen und kratzerartigen Oberhautabschürfungen. An der umgebenden Haut des Gesäßes fanden sich zahlreiche, z.T.
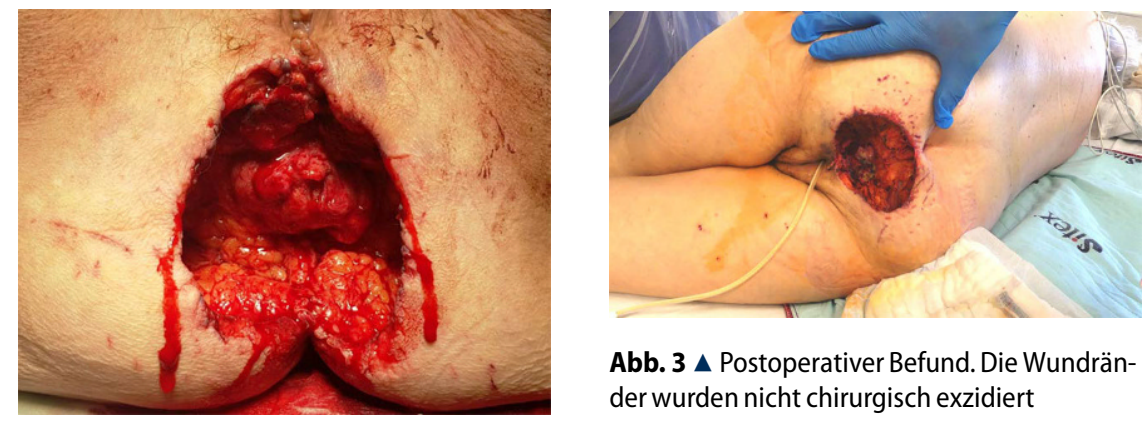

Abb. 3 A Postoperativer Befund. Die Wundränder wurden nicht chirurgisch exzidiert

Abb. $2 \Delta$ Präoperativer Befund in Steinschnittlage. (Quelle: Helios Klinikum Siegburg)

parallel verlaufende, längliche und punktförmige kratzerartige Hautverletzungen und an der rechten Oberschenkelrückseite mehrere punktförmige Verschorfungen (- Abb. 3 und 4).

Weitere Befunde der rechtsmedizinischen Untersuchung waren mehrere Hämatome an sturz- und anstoßtypischen Lokalisationen (u. a. Unterschenkelvorderseiten und Knie), ein gelb-bräunliches Hämatom an der linken Oberarmvorderseite am Übergang zum Brustkorb und am rechten Sprunggelenk innen eine rundliche, ringförmige, geformte, narbige Hautläsion, die an eine Kontaktverbrennung erinnerte. Es fanden sich keine typischen Abwehrverletzungen.

\section{Chemisch-toxikologische Untersuchungen}

Eine zum Zeitpunkt der Krankenhausaufnahme entnommenen Blutprobe wurde untersucht. Nachgewiesen wurden

- Zopiclon mit ca. $651 \mathrm{ng} / \mathrm{ml}$, (therapeutischer Bereich: $10-50 \mathrm{ng} / \mathrm{ml}$, toxischer Bereich: ab $150 \mathrm{ng} / \mathrm{ml}$, komatös-letaler Bereich: 600-1800 ng/ml [14]),

- eine Blutalkoholkonzentration von $0,48 \%$.

\section{Diskussion}

Hundebissverletzungen sind anhand des charakteristischen Abdruckmusters der Zähne und der Kombination von stumpfer mechanischer Druckschädigung des Gewebes und Perforationsverletzungen im Bereich der Reiß- und Fangzähne in der Regel gut zu identifizieren. Durch Scherbewegungen des Hundes und/oder des lebenden Opfers kann es dann zu tieferen und ausgedehnten Gewebeschädigungen kommen $[1,11]$.

Im Einzelfall können durch Hundebisse auch uncharakteristische Verletzungen entstehen, die zunächst nicht an Tierbisse denken lassen. Vor allem die Wundränder können dabei dann den entscheidenden Rückschluss auf die Traumatogenese zulassen. Als weiteres Kriterium für eine Verletzung durch Tierzähne können sich Kratzspuren von Tierkrallen an der umgebenden Haut finden [16].

In manchen Fällen kann das Verletzungsbild bei Angriffen von Hunden auf Lebende auch dem eines postmortalen Tierfraßes entsprechen, z. B. bei ausgedehnten Skalpierungsverletzungen oder Dekapitation von Kleinkindern durch Hunde $[15,17]$.

Postmortaler Tierfraß in häuslicher Umgebung stellt in der Rechtsmedizin kein unbekanntes Phänomen dar $[6,8,10]$ und Verletzungen abgrenzen. Fäulnisveränderungen können eine Unterscheidung zwischen vitaler, agonaler bzw. perimortaler und postmortaler Entstehung erschweren. Unter Umständen kann das Verletzungsbild auf den ersten Blick auch als Verletzungsbeibringung durch Dritte missinterpretiert werden.

Bei postmortalem Tierfraß sind in der Regel unbekleidete und leicht zugängliche Körperstellen wie das Gesicht und die Hände betroffen. Dabei kann es von einzelnen, fokalen Kratz- und Bissspuren bis hin zu ausgedehnten Defekten von Weichteil- und Knochengewebe oder Amputation ganzer Gliedmaßen oder des Kopfes kommen $[2,5]$. Selten finden sich Verletzungen von bekleideten Körperstellen, Eröffnung von Bauch- oder Brusthöhle oder lässt sich in der Regel gut von vitalen 
eine Verletzung der Genitalien [1, 3, 4, 7]. Charakteristisch sind unregelmäßige, fransige Wundränder mit kratzerartigen oder gezähnten Abschürfungen. Typischerweise entstehen diese durch das Einstechen der Eckzähne und anschließendes Reißen am Gewebe durch Kopfschütteln des Hundes, was durch das Körpergewicht der Leiche verstärkt werden kann. Werden die Wunden durch das Tier geleckt, können die Gewebe ein glänzendes, wie „poliertes" Aussehen annehmen [6].

Im vorgestellten Fall waren die oberflächlichen, linearen und fransigen Einkerbungen und Abschürfungen der Oberhaut an den Wundrändern charakteristisch für die Entstehung der Verletzung durch das Gebiss eines Hundes. An der Haut in unmittelbarer Nähe zu der Verletzung fanden sich zudem zahlreiche kratzerartige Läsionen durch Tierkrallen.

Aufgrund der chirurgischen Wundbehandlung waren molekularbiologische Untersuchungen zur Detektion von HundeDNA nicht mehr möglich. Auch eine Untersuchung des Hundes war nicht erfolgt, sodass die Ursache der Verletzung ausschließlich anhand der Wundmorphologie ermittelt werden musste.

In der Blutprobe der Geschädigten wurden zum Zeitpunkt der Krankenhausaufnahme eine toxische Zopiclonkonzentration und eine Alkoholkonzentration von $0,48 \%$ o nachgewiesen. Bei einer Liegezeit bis zu ca. $12 \mathrm{~h}$ ist davon auszugehen, dass zudem ein Teil der Substanzen zum Zeitpunkt der Blutentnahme bereits wieder abgebaut gewesen war. Demnach war es durch eine Mischintoxikation zu einer tiefen Bewusstlosigkeit mit aufgehobenem Schmerzempfinden gekommen, sodass gegen die Bissverletzungen des Tieres keinerlei Abwehrreaktionen der Geschädigten erfolgten. Durch das Ausbleiben von Gegenwehr lässt sich erklären, dass durch zahlreiche einzelne Bisseinwirkungen ein tiefer, gut begrenzter Weichteildefekt entstehen konnte, wie er sonst nur bei postmortalem Tierfraß zu sehen ist. Der Dackel hatte dabei nicht das gesamte Gewebe aus der Wunde gefressen, sondern auch Gewebereste in dem für ihn zugänglichen Bereich der Wohnung verteilt.

Die Motivation des Hundes bleibt letztlich nicht eindeutig geklärt. Nahrungsman- gel wird beispielsweise als Motivation diskutiert, wobei dieser jedoch bei Haustieren im Gegensatz zu Wildtieren eine eher untergeordnete Rolle spielt $[12,13]$. Im vorliegenden Fall ist nicht bekannt, ob der Dackel während des infrage kommenden Zeitraums auch Zugang zu Tierfutter gehabt hat.

Verstirbt der Hundebesitzer oder wird er bewusstlos, so ist das Tier mit einem ihm völlig unbekannten Verhalten seiner Bezugsperson konfrontiert. Auch der ihm bekannte Geruch kann sich verändert haben. In dieser Situation kann der Hund versuchen, die Aufmerksamkeit seines Besitzers zu erregen, indem er z. B. unbekleidete Körperbereiche leckt oder die Person mit seiner Schnauze anstößt. Wenn dadurch nicht die dem Tier bekannte und erwartete Reaktion hervorgerufen wird, kann es dazu kommen, dass der Hund zu beißen beginnt $[9,12,13]$. Aufgrund des unbekleideten Unterkörpers und einer Seitenlage der Geschädigten war der Anogenitalbereich für den Hund gut zugänglich. Denkbar ist auch, dass es im Rahmen der Bewusstlosigkeit zum Abgang von Stuhl und/oder Urin gekommen ist, was einen zusätzlichen Stimulus für das Tier dargestellt haben dürfte.

Die Frau konnte sich, bis auf dauerhafte Versorgung mit einem Anus praeter, gesundheitlich wieder vollständig erholen. Sowohl die Geschädigte als auch deren Ehemann waren seit Jahren alkoholabhängig. Sie stritten im weiteren Verlauf mehrfach vehement $a b$, dass die Verletzung durch den Dackel entstanden sein konnte, gaben aber auch keine alternative Traumatogenese an. Ob der Hund weiter im Haushalt des Ehepaares verblieb, ist nicht bekannt.

Bei dem hier vorgestellten Fall handelt es sich um den seltenen Befund von analem Tierfraß beim Lebenden. Anhand der Wundmorphologie, der Auffindesituation und der chemisch-toxikologischen Untersuchungen der Geschädigten konnte die Traumatogenese schlüssig erklärt werden.
Korrespondenzadresse

Inga Duval

Institut für Rechtsmedizin, Universitätsklinikum Bonn

Stiftsplatz 12, 53111 Bonn, Deutschland

inga.duval@ukbonn.de

Funding. Open Access funding enabled and organized by Projekt DEAL.

\section{Einhaltung ethischer Richtlinien}

Interessenkonflikt. I. Duval, E. Doberentz und B. Madea geben an, dass kein Interessenkonflikt besteht.

Es wurden keine Studien an Tieren und Menschen durchgeführt. Die Untersuchungen erfolgten unter Einhaltung der Vorgaben der Zentralen Ethikkommission der Bundesärztekammer. Es liegen im Manuskript keine Bilder oder andere Angaben vor, anhand derer der Patient zu identifizieren ist.

Open Access. Dieser Artikel wird unter der Creative Commons Namensnennung 4.0 International Lizenz veröffentlicht, welche die Nutzung, Vervielfältigung, Bearbeitung, Verbreitung und Wiedergabe in jeglichem Medium und Format erlaubt, sofern Sie den/die ursprünglichen Autor(en) und die Quelle ordnungsgemäß nennen, einen Link zur Creative Commons Lizenz beifügen und angeben, ob Änderungen vorgenommen wurden.

Die in diesem Artikel enthaltenen Bilder und sonstiges Drittmaterial unterliegen ebenfalls der genannten Creative Commons Lizenz, sofern sich aus der Abbildungslegende nichts anderes ergibt. Sofern das betreffende Material nicht unter der genannten Creative Commons Lizenz steht und die betreffende Handlung nicht nach gesetzlichen Vorschriften erlaubt ist, ist für die oben aufgeführten Weiterverwendungen des Materials die Einwilligung des jeweiligen Rechteinhabers einzuholen.

Weitere Details zur Lizenz entnehmen Sie bitte der Lizenzinformation auf http://creativecommons.org/ licenses/by/4.0/deed.de.

\section{Literatur}

1. Bury D, Langlois N, Byard RW (2012) Animal-related fatalities - part I: characteristic autopsy findings and variable causes of death associated with blunt and sharp trauma. J Forensic Sci 57(2):370-374. https://doi.org/10.1111/j.1556-4029.2011.01921.

2. Buschmann C, Solarino B, Püschel K, Czubaiko F, Heinze S, Tsokos M (2011) Post-mortem decapitation by domestic dogs: three case reports and review of the literature. Forensic Sci Med Pathol 7(4):344-349. https://doi.org/10.1007/s12024011-9233-x

3. Buschmann C, Wrobel D, Tsokos M (2008) Postmortem animal depredation of the genital region caused by a half-breed dog. Arch Kriminol 222(5-6):182-186 
4. Byard RW, James RA, Gilbert JD (2002) Diagnostic problems associated with cadaveric trauma from animal activity. Am J Forensic Med Pathol 23(3):238-244. https://doi.org/10.1097/ 00000433-200209000-00006

5. Chute DJ, Bready RJ (2017) A case of postmortem canine depredation. Am J Forensic Med Pathol 38(2):100-102. https://doi.org/10.1097/ PAF.0000000000000303

6. Colard T, Delannoy Y, Naji S, Gosset D, Hartnett K, Bécart A (2015) Specific patterns of canine scavenging in indoor settings. J Forensic Sci 60(2):495-500. https://doi.org/10.1111/15564029.12684

7. Driever F, Dettmeyer R, Madea B (2003) An unusual area of injury in postmortem animal feeding beneath clothing. Arch Kriminol 211(1-2):27-32

8. Karger B, Rothschild MA (2004) Postmortaler Tierfraß durch Wirbeltiere. In: Brinkmann B, Madea B (Hrsg) Handbuch gerichtliche Medizin, Bd. 1. Springer, Berlin Heidelberg New York, S187-191

9. Hernández-Carrasco M, Pisani JMA, ScarsoGiaconi F, Fonseca GM (2016) Indoor postmortem mutilation by dogs: confusion, contradictions and needs from the perspective of the forensic veterinarian medicine. J Vet Behav 15:56-60. https://doi.org/10.1016/j.jveb.2016.08.074

10. Rossi ML, Shahrom AW, Chapman RC, Vanezis P (1994) Postmortem injuries by indoor pets. Am J Forensic Med Pathol 15(2):105-109. https://doi. org/10.1097/00000433-199406000-00004

11. Rothe K, Tsokos M, Handrick W (2015) Animal and human bite wounds. Dtsch Arztebl Int 112(25):433-442. https://doi.org/10.3238/ arztebl.2015.0433

12. Rothschild MA, Schneider V (1997) On the temporal onset of postmortem animal scavenging "Motivation" of the animal. Forensic Sci Int 89(1-2):57-64. https://doi.org/10.1016/s03790738(97)00112-6

13. Schumann M, Nolte I, Huckenbeck W, Barz J (1996) Tierfraß - wenige Stunden nach Todeseintritt? Rechtsmedizin 7:22-24. https://doi.org/10.1007/ BF03042336

14. Schulz M, Iwersen-Bergmann S, Andresen $H$, Schmoldt A (2012) Therapeutic and toxic blood concentrations of nearly 1,000 drugs and other xenobiotics. Crit Care 16:R136

15. Tsokos M, Byard RW, Püschel K (2007) Extensive and mutilating craniofacial trauma involving defleshing and decapitation: unusual features of fatal dog attacks in the young. Am J Forensic Med Pathol 28(2):131-136. https://doi.org/10.1097/01. paf.0000257395.90724.39

16. Tsokos M, Schulz F (1999) Indoor post-mortem animal interference by carnivores and rodents: report of two cases and review of the literature. Int J Legal Med 112(2):115-119. https://doi.org/10. 1007/s004140050212

17. Tsokos M, Schulz F, Püschel K (1999) Unusual injury pattern in a case of postmortem animal depredation by a domestic German shepherd. Am J Forensic Med Pathol 20(3):247-250. https://doi. org/10.1097/00000433-199909000-00006

\section{Vital anal mutilation by a dachshund}

Dog bite injuries in the living usually show a characteristic morphology that easily indicates the trauma mechanism. If dogs have access to a corpse, postmortem depredation injuries can occur and the pattern of injury can at first glance be misinterpreted as being caused by third parties.

We present the rare case of a premortem animal depredating injury to a living person. Due to unconsciousness caused by a mixed intoxication with alcohol and zopiclone, bite injuries of the owner's dachshund led to an extensive tissue defect in the anal region. Since neither swabs of the wound nor an examination of the dog were available, the cause of injury had to be determined solely on the basis of the wound morphology.

\section{Keywords}

Dog bite $\cdot$ Animal predation · Bite injury $\cdot$ Anal trauma $\cdot$ Zopiclone 